\title{
Prevalence and characteristics of Alice in Wonderland Syndrome in adult migraineurs: Perspectives from a tertiary referral headache unit
}

Cephalalgia

$0(0) 1-10$

(C) International Headache Society 2020

Article reuse guidelines:

sagepub.com/journals-permissions DOI: $10.1177 / 0333102420968245$ journals.sagepub.com/home/cep @SAGE

\author{
Giulio Mastria ${ }^{1,2}$, , Valentina Mancini ${ }^{1,3} *$ (D), Marco Di Cesare', \\ Marta Puma', Michele Alessiani', Barbara Petolicchio', \\ Alessandro Viganò ${ }^{4, \#}$ and Vittorio Di Piero ${ }^{1,5, \#}$
}

\begin{abstract}
Background: Migraine affects how the brain processes sensory information at multiple levels. The aberrant integration of visual and somatosensory stimuli is thought to underlie Alice in Wonderland Syndrome, a disorder often reported as being associated with migraine. However, there is still a lack of knowledge about the epidemiology of this syndrome in migraineurs and the association between Alice in Wonderland Syndrome episodes and migraine attacks. Therefore, we conducted a prospective cohort study to systematically evaluate the prevalence and the clinical features of Alice in Wonderland Syndrome in a large sample of patients with migraine.

Methods: All the patients attending for the first time a tertiary-level headache clinic were consecutively screened for Alice in Wonderland Syndrome symptoms by means of an ad hoc questionnaire and detailed clinical interview, over a period of 1.5 years. Patients experiencing Alice in Wonderland Syndrome symptoms were contacted for a follow-up after 8-12 months.

Results: Two hundred and ten patients were recruited: 40 patients (19\%) reported lifetime occurrence of Alice in Wonderland Syndrome, $90 \%$ of whom (38/40) had migraine with aura. Thirty-one patients experienced episodes of Alice in Wonderland Syndrome within I h from the start of migraine headache. Patients reported either visual or visual and somatosensory symptoms (i.e. somatosensory symptoms never presented alone). We collected the follow-up details of 30 patients with Alice in Wonderland Syndrome, 18 of whom had been prescribed a preventive treatment for migraine. After 8-12 months, 5 of the treated patients reported a decrease, while 13 reported no episodes of Alice in Wonderland Syndrome.

Conclusion: Alice in Wonderland Syndrome prevalence in migraineurs was found to be higher than expected. Alice in Wonderland Syndrome was mostly associated with migraine with aura and tended to occur close to the migraine attack, suggesting the existence of a common pathophysiological mechanism. Patients treated with migraine preventive treatments had a higher chance of decreasing or even resolving Alice in Wonderland Syndrome episodes.
\end{abstract}

\section{Keywords}

AIWS, migraine aura, body representation, cortical spreading depression, migraine preventive treatment

Date received: II May 2020; accepted: 2I September 2020

\footnotetext{
'Department of Human Neurosciences, Sapienza - University of Rome, Rome, Italy

${ }^{2}$ My Space Lab, Department of Clinical Neuroscience, Centre Hospitalier Universitaire Vaudois (CHUV), University of Lausanne, Lausanne, Switzerland

${ }^{3}$ Developmental Imaging and Psychopathology Laboratory, University of Geneva School of Medicine, Geneva, Switzerland

${ }^{4}$ IRCCS Fondazione Don Carlo Gnocchi, Milan, Italy

${ }^{5}$ University Consortium for Adaptive Disorders and Head Pain UCADH, Pavia, Italy
}

\footnotetext{
*These authors contributed equally to this work.

\#These authors are shared last authors

Corresponding author:

Valentina Mancini, Università degli Studi di Roma La Sapienza, Viale dell'Università 30, Roma, 00185, Italy.

Email: v.mancini92@gmail.com
} 


\section{Introduction}

Most recent accounts of migraine pathophysiology highlight that migraine is not only a pain disorder, but rather a brain state that affects multiple mechanisms involved in the processing and filtering of sensory stimuli (1). Consistently, the clinical presentation of migraine is remarkably complex and multifaceted, comprising unisensory and higher-order multi-sensory disturbances $(2,3)$. Some of these symptoms have been found to be far more frequent in migraineurs than in the general population and, therefore, should be considered migraine-traits (2). However, these peculiar migraine phenotypes are not systematically taken into account by the current International Classification of Headache Disorders, 3rd edition (ICHD-3) (4). A practical reason for the absence of these symptoms from ICHD-3 is their relative rarity and the lack of studies aimed at investigating their relationship with migraine itself.

In this regard, one notable example is Alice in Wonderland Syndrome (AIWS), a syndrome characterised by visual and somatosensory misperceptions (5-7). The association between AIWS and migraine is mostly anecdotal, since it has been investigated only by case reports or small studies (8-15). Moreover, while migraine is the first cause of AIWS in adults $(6,16)$, most of the studies on AIWS have focused on children $(6,17,18)$. A follow-up study reported that the prevalence of AIWS was around $18 \%$ in a cohort of 28 pediatric patients with migraine (19). Likewise, a recent retrospective study limited to vestibular migraine found 17 AIWS cases out of 121 patients (14\%) (20). However, to the present time, no study has systematically evaluated the prevalence of AIWS in adult migraineurs.

Another critical aspect is the lack of agreement on AIWS diagnosis criteria (18). Patients experiencing episodes of AIWS may feel that parts of their body are bigger (macrosomatognosia) or smaller (microsomatognosia). They might also have an aberrant visual perception, seeing objects either as smaller or bigger (micropsia/macropsia), closer or further away (pelopsia/telopsia) than in reality $(5,7,21)$. These visual and somatosensory disturbances are generally considered core symptoms of AIWS $(5,7,17,21)$. Derealisation, depersonalisation and slowing of the perception of time have also been reported in the literature (21). However, many independent groups have defined them as "facultative" symptoms; namely, they are not specific to the syndrome and not sufficient for a diagnosis of AIWS $(16,22)$. Additionally, these dissociative symptoms might complicate and delay the diagnosis, since they have a strong association not only with migraine but also with other neurological and psychiatric diseases (23-25).

Aside from epidemiology, several other questions concerning AIWS remain unanswered. To date, the temporal association of AIWS episodes with migraine attacks has not yet been investigated. On the one hand, the relationship between AIWS and migraine might be explained in the context of a general susceptibility of migraineurs to experience complex sensory and neuropsychological symptoms (2). This seems to be the case in children, where no temporal association between the AIWS episodes and migraine attacks have been clearly shown (18). On the other hand, this relationship might be more specific in adult migraineurs. On the basis of some previous reports, we investigated the hypothesis that, in this population, AIWS symptoms might be compatible in terms of onset and duration with the clinical features of migraine aura.

Moreover, AIWS is often reported to be associated with psychiatric conditions, especially depressive and anxiety disorders $(26,27)$, which are highly prevalent among migraineurs and are known to have a strong impact on migraine phenotype (28-30). In patients with migraine, these disturbances might have a role in the occurrence of AIWS.

Beyond its theoretical value, defining the precise relationship between AIWS and migraine might inform an appropriate therapy, especially in the presence of particularly distressful symptoms. If AIWS and migraine attacks share, at least in part, the same pathophysiology, the drugs used for migraine prevention might have an effect on AIWS, as previously shown in patients with vestibular migraine (20).

In summary, the aim of the present study is to systematically evaluate the prevalence of AIWS in a sample of consecutive patients with migraine and try to answer these clinical questions in order to improve the ability to diagnose and treat patients with migraine and episodes of AIWS.

\section{Methods}

\section{Study design and recruitment of patients}

We conducted a prospective cohort study in the Headache Center of Policlinico Umberto I in Rome. Over a period of 18 months, all patients who attended our clinic for the first time, diagnosed with migraine according to ICHD-3 and naïve for preventive therapies, were consecutively screened for symptoms of AIWS via an ad hoc questionnaire.

During the visit, all patients underwent a comprehensive clinical interview according to ICHD-3 to obtain a headache diagnosis. The age at onset of migraine, migraine type and the average frequency of 
migraine attacks per month during the 3 months prior to the visit were recorded.

Past clinical history of neurological and psychiatric comorbidities as well as ongoing or prior pharmacological treatments were collected by trained neurologists. Specifically, the presence of major depressive and anxiety disorders was based on a previous diagnosis and/or related pharmacological treatment. All patients underwent a complete neurological examination.

When appropriate, a migraine preventive treatment was prescribed by the treating neurologist, regardless of the diagnosis of AIWS. The main factors influencing the choice of the class of medication were age, migraine characteristics, comorbidities and drug-to-drug interactions.

After the visit, the answers to the questionnaire were double-checked by two trained physicians, who directly interviewed participants and collected information about AIWS and migraine characteristics. Clinical characteristics of AIWS episodes were explored, such as the age of onset and the temporal association between AIWS, migraine and aura. In detail, the temporal association was defined as a categorical variable. AIWS was considered temporally associated with migraine if it occurred from $1 \mathrm{~h}$ prior to pain onset to pain resolution (either spontaneous or druginduced). Otherwise, AIWS and migraine attacks were considered temporally unrelated. AIWS was defined as active if episodes compatible with AIWS occurred in the 3 months prior to the consultation. The rate of self-reported AIWS was estimated by comparing the answers to the questionnaires and the records of the visit. Structural brain MRI and EEG were performed in all the patients with AIWS in order to rule out other neurological conditions, as recommended by Valença and colleagues (18).

Patients with AIWS symptoms at the time of the enrollment were followed up for 8-12 months in order to assess the presence of AIWS episodes during the period of treatment. In the context of a detailed follow-up interview, we quantified the benefit of migraine preventive treatment on AIWS symptoms by using the categories of $100 \%, 50 \%, 30 \%$ and $0 \%$ reduction of the frequency of AIWS episodes.

\section{Definition of Alice in Wonderland Syndrome}

In agreement with previous literature, only patients presenting a somatosensory and/or visual disturbance were diagnosed with AIWS $(16,17)$. Specifically, the questionnaire investigated the presence of AIWS according to the classification proposed by Liu and colleagues, which makes a distinction between somatosensory and visual disturbances alone (respectively
AIWS type A and B) or together (type C) (17). On the other hand, dissociative symptoms (e.g. slowing in the perception of time, depersonalisation and derealisation) were recorded but were not used to identify an episode of AIWS if occurring alone $(16,22)$.

Standard protocol approvals, registrations, and patient consents. All subjects gave written informed consent. This study was approved by the Ethics Committee of Human Experimentation of Policlinico Umberto I University Hospital and conformed to the latest version of the declaration of Helsinki.

Statistics. No statistical power calculation was conducted before the study and the sample size was based on all the available data. For analysis, groups were defined by the diagnosis of AIWS, migraine with aura (MA) and migraine without aura (MoA), with each patient belonging to only one group. A simple linear regression model was used to test differences between the group of patients with AIWS and the two groups of patients without AIWS. Age at the time of recruitment, age at migraine onset, frequency and duration were used as dependent variables, while the diagnosis (i.e. AIWS, MA, MoA) was used as an explanatory variable (e.g. age $\sim$ diagnosis). In the case of migraine frequency, age and sex were added to the model as covariates. Similarly, logistic regression models were used to compare the presence of facultative symptoms of AIWS (depersonalisation, derealisation and slowing in time perception), psychiatric comorbidities (e.g. depressive and anxiety disorders) and the drug of choice for preventive treatment of migraine in the three groups. Age and sex were introduced as covariates to take into account possible differences between the three groups.

Chi-squared tests were used to compare the prevalence of female sex between groups; $95 \%$ confidence intervals $(\mathrm{CI})$ for each prevalence rate were calculated with the Wilson score interval. All the statistical analyses were conducted with the $\mathrm{R}$ statistical package, version 3.5.3, using a significant level of $p<0.05$ Bonferroni corrected.

\section{Results}

Out of 223 migraine patients visiting for the first time in our center, 210 questionnaires were collected over 18 months. The remaining 13 patients refused to participate. Out of the 170 patients without AIWS, 118 (44\%, CI: $35-53)$ received a diagnosis of MoA, $52(25 \%$, CI: 19-31) of MA with typical aura. No patient had hemiplegic migraine. The remaining 40 patients with migraine experienced episodes of AIWS (see Figure 1), and while 38 of 40 patients with AIWS 


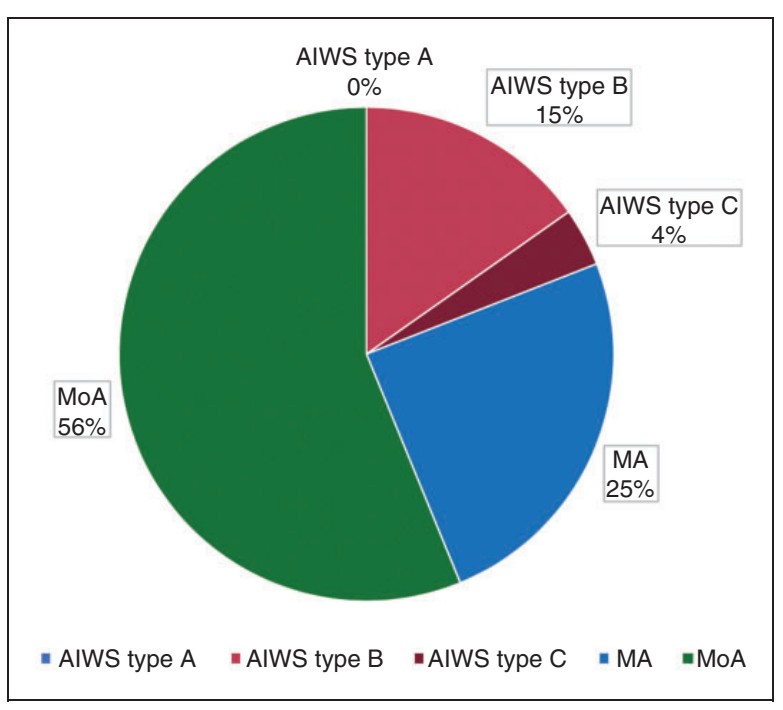

Figure I. The pie graph shows the distribution of migraine without aura (MoA), migraine with aura (MA) and Alice in Wonderland Syndrome (AIWS) in our sample.

AIWS: Alice in Wonderland Syndrome; MA: migraine with aura; MoA: migraine without aura.

had MA, only two were diagnosed with MoA. The prevalence of AIWS in our headache unit was around 19\% (CI: 14-25). Only four patients (10\%, CI: 4-23) spontaneously reported AIWS symptoms during the visit. Thirty-two patients (80\%, CI: $65-90)$ had only visual symptoms, while the remaining $8(20 \%$, CI: 10-35) patients had both somatosensory and visual symptoms; no patient had isolated somatosensory symptoms (see Figure 1). The EEG and MRI of all the patients with AIWS were unremarkable. No patient with AIWS reported illicit use of drugs.

Demographic information of the three groups are reported in Table 1. No sex differences were observed across groups (AIWS vs. MA: chi-squared $=1.71$, $p=0.191 ; \quad$ AIWS vs. MoA chi-squared $=0.24$, $p=0.625$ ). Four patients experienced AIWS during their childhood. In one case the age of onset of migraine and AIWS was the same, while in three cases AIWS episodes started earlier. Patients with AIWS were on average younger than patients with $\mathrm{MA}$ and MoA (age $\sim$ diagnosis, $\mathrm{F}=5.61, p=0.004$; $\left.\beta_{\mathrm{MA}}=7.73, p_{\mathrm{MA}}=0.013 ; \beta_{\mathrm{MoA}}=8.89, p_{\mathrm{MoA}}=0.001\right)$, had an earlier onset of migraine with respect to patients with MoA but not compared to those with MA (onset $\sim$ diagnosis, $\mathrm{F}=4.25, \quad p=0.015 ; \quad \beta_{\mathrm{MoA}}=6.55$, $\left.p_{\mathrm{MoA}}=0.009 ; \beta_{\mathrm{MA}}=2.47, p_{\mathrm{MA}}=0.382\right)$ and had no statistically different duration of migraine attacks compared to the other groups (duration $\sim$ diagnosis, $\mathrm{F}=1.27, \quad p=0.283 ; \quad \beta_{\mathrm{MA}}=4.94, \quad p_{\mathrm{MA}}=0.128$; $\beta_{\mathrm{MoA}}=2.03, p_{\mathrm{MoA}}=0.476$ ) (see Figure 2 ). Moreover, we found that chronic migraine, as defined by ICHD-3, was less frequent among patients with AIWS (25\%) than among those with MA and MoA (respectively $53 \%$ and $65 \%$ ). Migraine patients with AIWS had on average 8 days of headache per month vs. 19 days in patients with MA and 17 in patients with MoA (frequency $\sim$ diagnosis + age + sex, $\mathrm{F}=10.26, \quad p<0.001$; $\left.\beta_{\mathrm{MA}}=9.89, p_{\mathrm{MA}}<0.001 ; \beta_{\mathrm{MoA}}=8.64, p_{\mathrm{MoA}}<0.001\right)$.

The three groups did not differ in terms of depressive symptoms, but patients with AIWS had a lower burden of anxiety disorders (anxiety $\sim$ diagnosis + age + sex: $\quad p<0.018 ; \quad \beta_{\mathrm{MA}}=1.63$, $p_{\mathrm{MA}}<0.001 ; \beta_{\mathrm{MoA}}=1.53, p_{\mathrm{MoA}}<0.010$ ) (Figure 3, panel (a)).

Preventive treatments for migraine tended to be more often prescribed to patients with AIWS. Specifically, antiepileptics were more common in AIWS compared to MoA but not compared to MA (antiepileptics $\sim$ diagnosis + age + sex: $\quad \beta_{\mathrm{MA}}=-1.14$, $\left.p_{\mathrm{MA}}=0.068 ; \beta_{\mathrm{MoA}}=-1.71, p_{\mathrm{MoA}}=0.003\right)$ (Figure 3, panel (b)).

Regarding dissociative symptoms often associated with AIWS, only depersonalisation (depersonalisation $\sim$ diagnosis + age + sex: $\quad \beta_{\mathrm{MA}}=\quad-1.73$, $\left.p_{\mathrm{MA}}<0.001 ; \beta_{\mathrm{MoA}}=-2.81, p_{\mathrm{MoA}}<0.010\right)$ and derealisation (derealisation $\sim$ diagnosis + age + sex: $\quad \beta_{\mathrm{MA}}=$ $\left.-1.3, p_{\mathrm{MA}}<0.013 ; \beta_{\mathrm{MoA}}=-2.25, p_{\mathrm{MoA}}<0.001\right)$ were more frequent in the group of AIWS with respect to patients with MA and with MoA (Figure 3, panel (c)). On the other hand, the frequency of slowing in time perception was similar between subjects with AIWS and with MA, but differed with respect to the group with MoA (slowing in time perception $\sim$ diagnosis + age $+\operatorname{sex} \quad \beta_{\mathrm{MA}}=-0.67, \quad p_{\mathrm{MA}}=$ $\left.0.129 ; \beta_{\mathrm{MoA}}=-2.41, p_{\mathrm{MoA}}<0.001\right)$. For the results reported, age and sex did not have a significant effect on the variables tested.

Finally, episodes of AIWS started on average 3 years before the onset of migraine, consistently with previous studies (18). More than $90 \%$ of patients had an active AIWS at the recruitment.

Regarding the temporal association between AIWS and migraine, we found that the vast majority of AIWS patients experienced episodes of AIWS in concomitance with migraine attacks (Table 2). Specifically, $77.5 \%(31 / 40)$ of all the patients with AIWS and $81.6 \%(31 / 38)$ of the patients with AIWS and a diagnosis of migraine with aura reported a temporal association with migraine attacks (from $60 \mathrm{~min}$ prior to pain onset to pain resolution). The mean duration of AIWS episodes was $40 \mathrm{~min}$ (range: $10-60 \mathrm{~min}$ ).

\section{Follow-up}

At the time of enrolment, only three out of 40 patients with a history of AIWS did not have active AIWS. 
Table I. Demographic and clinical information of the three groups. Mean and standard deviation and frequency counts (percentage) are reported.

\begin{tabular}{llll}
\hline & AIWS & MA & MoA \\
\hline Number of patients & $19 \%(n=40)$ & $25 \%(n=52)$ & $56 \%(n=118)$ \\
Sex (female) & $85 \%(n=34)$ & $76.9 \%(n=40)$ & $82.2 \%(n=97)$ \\
Mean age (years) & $39 \pm 14$ & $47 \pm 13$ & $47 \pm 15$ \\
Mean age at onset & $19 \pm 10$ & $23 \pm 12$ & $26 \pm 14$ \\
Years of disease & $19.7 \pm 12.6$ & $24.2 \pm 13.9$ & $21.7 \pm 15.6$ \\
Days of migraine/month & $8.1 \pm 6.7$ & $19.7 \pm 8.3$ & $17.4 \pm 9.1$ \\
Chronic migraine (\%) & $25 \%(n=10)$ & $53 \%(n=28)$ & $65 \%(n=77)$ \\
Derealisation & $28.8 \%(n=15)$ & $15.4 \%(n=8)$ & $6.8 \%(n=8)$ \\
Depersonalisation & $50 \%(n=20)$ & $13.5 \%(n=7)$ & $5.1 \%(n=6)$ \\
Slowing in time perception & $57.5 \%(n=23)$ & $38.5 \%(n=20)$ & $10.2 \%(n=12)$ \\
Depression & $15 \%(n=6)$ & $13.5 \%(n=7)$ & $14.4 \%(n=17)$ \\
Anxiety & $7.5 \%(n=3)$ & $26.9 \%(n=14)$ & $24.6 \%(n=29)$ \\
Preventive therapy & $50 \%(n=20)$ & $28.8 \%(n=15)$ & $39.8 \%(n=47)$ \\
\hline
\end{tabular}

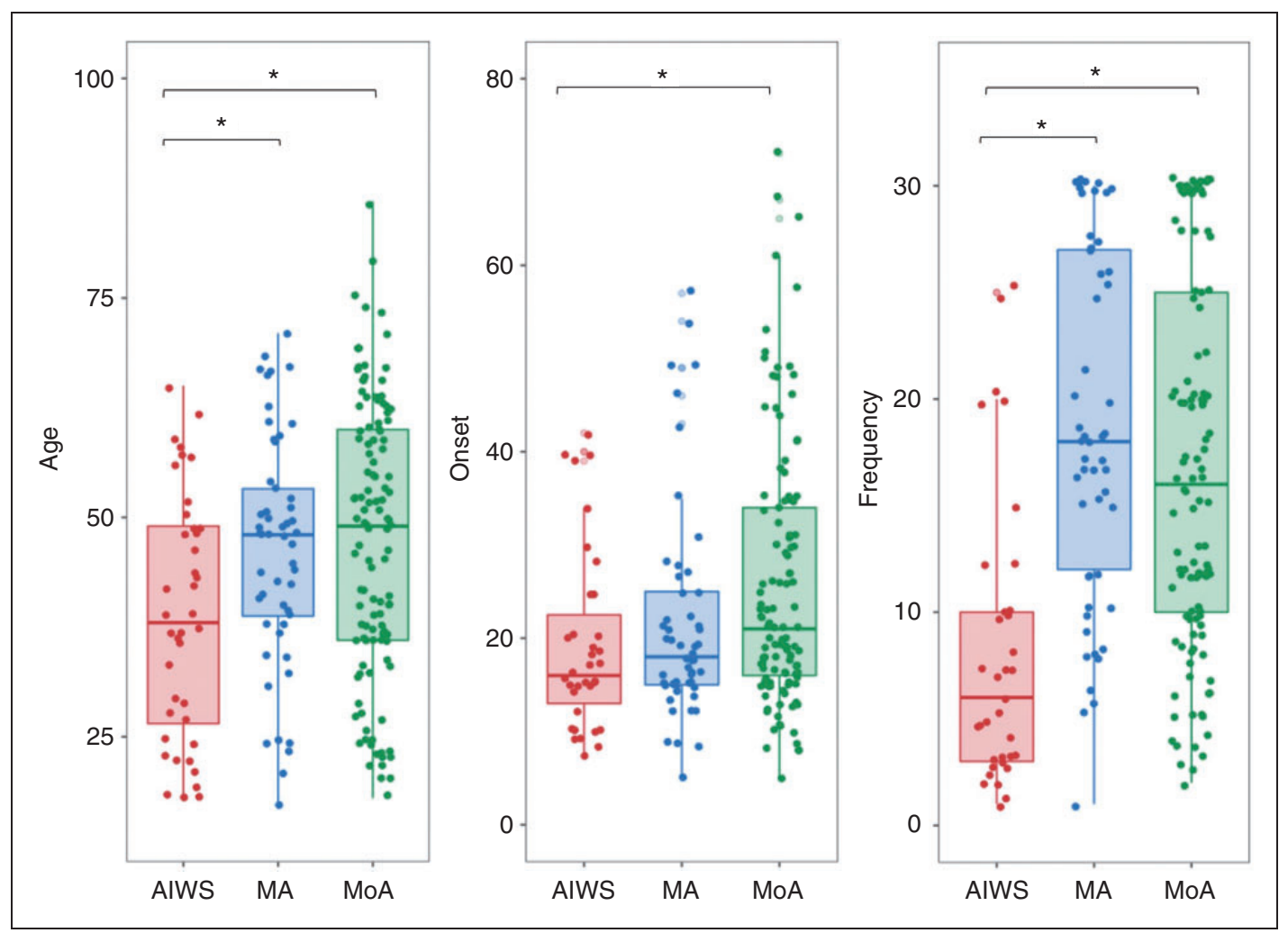

Figure 2. The box plots show the median and the $95 \% \mathrm{Cl}$ of age, the age of onset and the frequency of migraine in the three groups. Patients with AIWS are younger and have a lower number of migraine attacks per month.

*p-value $<0.05)$.

MA: migraine with aura; MoA: migraine without aura.

The remaining 37 patients were followed up for 8 to 12 months. Seven patients refused to participate or dropped out. Eighteen patients were treated with pharmacological preventive treatment (nine with antiepileptics, four with antidepressants, five with beta-blockers), two were following an experimental short-term psychodynamic psychotherapy (STPP) $(31,32)$, and 10 were not receiving any preventive treatment. While the vast majority of patients who did not receive any preventive treatment $(80 \%, \mathrm{CI}: 49-94)$ and all those who did STPP did not experience any benefit to AIWS, all patients treated with pharmacological prophylaxis had a reduction in AIWS episodes: 13 (72\%, CI: $51-$ $86)$ had $100 \%$ response, and five (28\%, CI: $14-49)$ had 


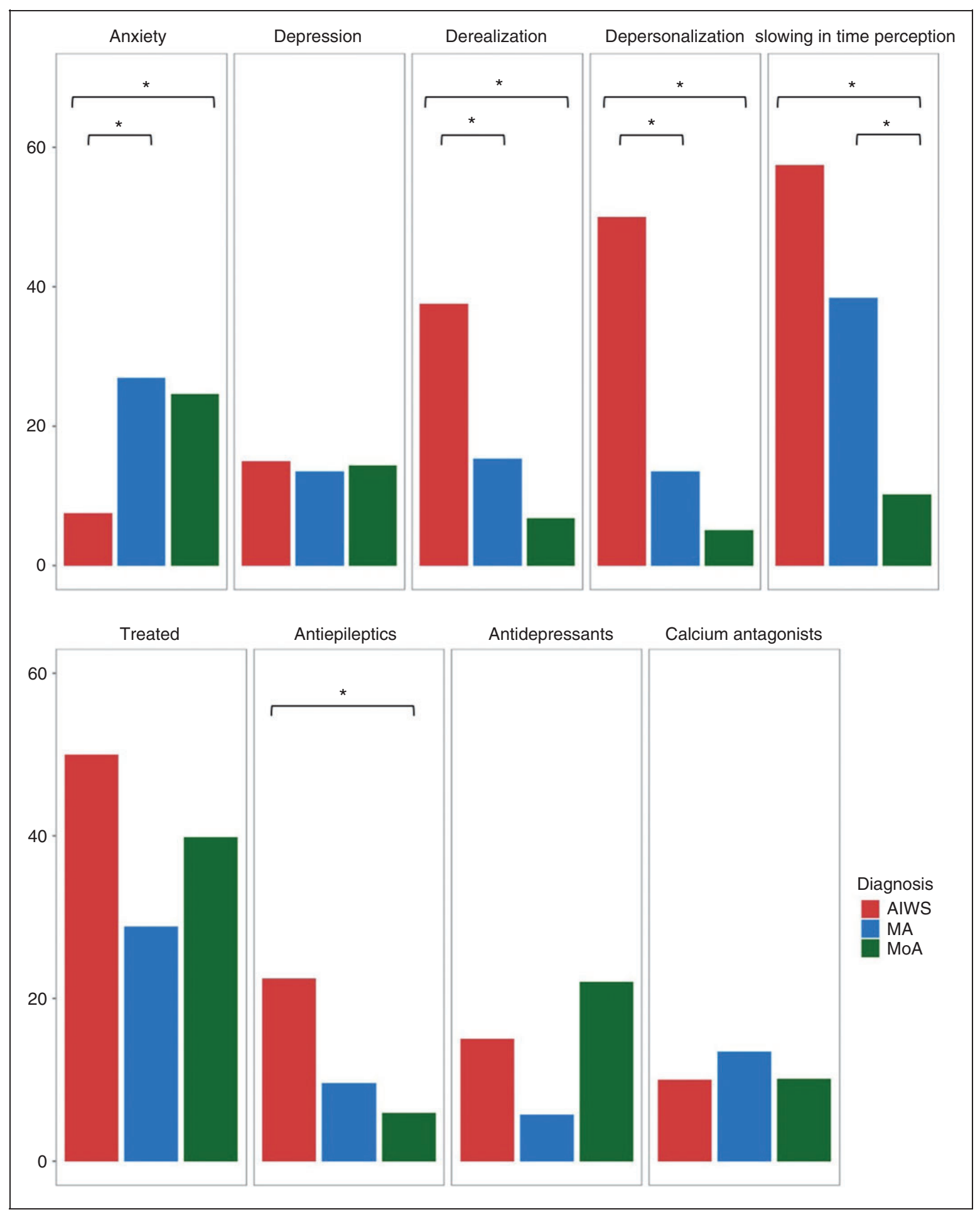

Figure 3. Upper panel: The first two bar plots show the prevalence (prevalence of subject presenting etc.) of anxiety and depression in our sample. Anxiety is less common among patients with AIWS than in those with MA or MoA, while depression is equally prevalent in the three groups. The last bar plots show the prevalence of AIWS facultative symptoms. These symptoms are more frequent but not exclusive to patients with AIWS. Lower panel: Patients with AIWS are more often prescribed with a preventive therapy and antiepileptics are far more frequently administered in this group of patients than in patients with simple MA or MoA. $*_{p}$-value $\left.<0.05\right)$. All the results are covaried for sex and age. 
Table 2. Clinical characteristics of AIWS episodes. Mean and standard deviation and frequency counts (percentage) are reported.

\begin{tabular}{ll}
\hline Mean age at onset (migraine) & $16 \pm 10$ (range: 7-40) \\
Mean age at onset (AIWS episodes) & $19 \pm 10$ (range: $7-45)$ \\
Mean duration of AIWS episodes & 40.3 min (range: 5 min to $4 \mathrm{~h}$ ) \\
Temporal association with migraine aura & $77.5 \%(\mathrm{n}=31)$ \\
Active AIWS & $92.5 \%(\mathrm{n}=37)$ \\
\hline
\end{tabular}

Table 3. Percentage of distinct AIWS symptoms endorsed by the patients.

\begin{tabular}{ll}
\hline AIWS symptoms & $\begin{array}{l}\text { Number of } \\
\text { patients (\%) }\end{array}$ \\
\hline Macrosomatognosia & $3(7.5 \%)$ \\
Microsomatognosia & $5(12.5 \%)$ \\
Other alterations of body schema* & $4(10 \%)$ \\
Micropsia & $15(37.5 \%)$ \\
Macropsia & $15(37.5 \%)$ \\
Telopsia & $20(50 \%)$ \\
Pelopsia & $19(47.5 \%)$ \\
More than one symptom & $36(90 \%)$ \\
AIWS facultative symptoms & \\
Derealisation & $15(37.5 \%)$ \\
Depersonalisation & $16(40 \%)$ \\
Slowing in time perception & $18(45 \%)$ \\
Other visual symptoms reported & \\
Mosaic vision & $20(50 \%)$ \\
Kinetopsia & $3(7.5 \%)$ \\
2D-3D inversion & $1(2.5 \%)$ \\
Synesthesia & $2(5 \%)$ \\
\hline
\end{tabular}

*The term "other alterations of body schema" refers to transient asomatognosia reported by the patients, i.e. the loss of recognition of part of their own body.

more than $50 \%$ response (see flow chart, Figure 4). No patient experienced a $30 \%$ decrease or no reduction at all.

\section{Discussion}

Although AIWS is often considered a rare condition, our results show that ad hoc questions targeting AIWS symptoms at the first visit can reveal a prevalence of around $19 \%$ in a tertiary referral headache unit. This result is in line with previous reports in children with migraine and slightly higher than that found in vestibular migraine (20). In this regard, the paper by Jurgens and colleagues showed that the prevalence of some complex sensory disturbances is higher among migraineurs than in the general population (2). Interestingly, in $90 \%$ of cases, patients answered positively to the items related to AIWS symptoms in the questionnaire, but only $10 \%$ spontaneously reported the symptoms to the neurologist during the visit. The fact that these symptoms can be uncanny or even distressful raises an important question: Why do patients not spontaneously complain about them with their treating physicians, so that such conditions are considered rare cases even in specialised literature? Probably, patients hardly perceived AIWS symptoms as related to migraine, but rather they fear that such disturbances could be interpreted as a form of mental illness and, therefore, liable to stigmatisation. In our experience, understanding the origin of these symptoms helps the patients to cope with the disease better. Therefore, it is up to the treating physician to include specific questions about AIWS during the collection of the medical history, even though it is not always simple to obtain this information in the short time of an outpatient visit, in clinics with a high attendance of patients.

Despite the fact that AIWS has been historically associated with migraine in general $(5,18)$, our findings highlighted that the vast majority of the patients with AIWS (95\%) received a diagnosis of MA, while only $5 \%$ had MoA. In agreement with previous reports, the visual form of AIWS seems to be the most prevalent, followed by visual-somatosensory forms $(16,17)$. However, in our series, we did not find any patient reporting isolated alterations of the body representation. The complete absence of AIWS presenting with only somatosensory symptoms in our sample is inconsistent with a recent work showing a higher proportion of somesthetic distortions in patients with vestibular migraine (20). However, in our study, we found only one patient fulfilling the criteria for vestibular migraine (4), making the two results not directly comparable.

Although this is an epidemiological study, our results seem to point towards a possible pathophysiological relationship between AIWS and migraine aura. AIWS mostly occurs in MA patients and presents with visual alterations in all episodes. It often precedes migraine attacks, when aura symptoms are most likely to occur. In MA, aura symptoms are thought to be caused by the propagation of Cortical Spreading Depression (CSD), a neural event that seems to be an intrinsic property of the migrainous brain (33). It is conceivable that in some patients with MA, CSD might affect higher-order associative cortices, leading to various degrees of visual and somatosensory integration disturbances, as we reported in one single case. 


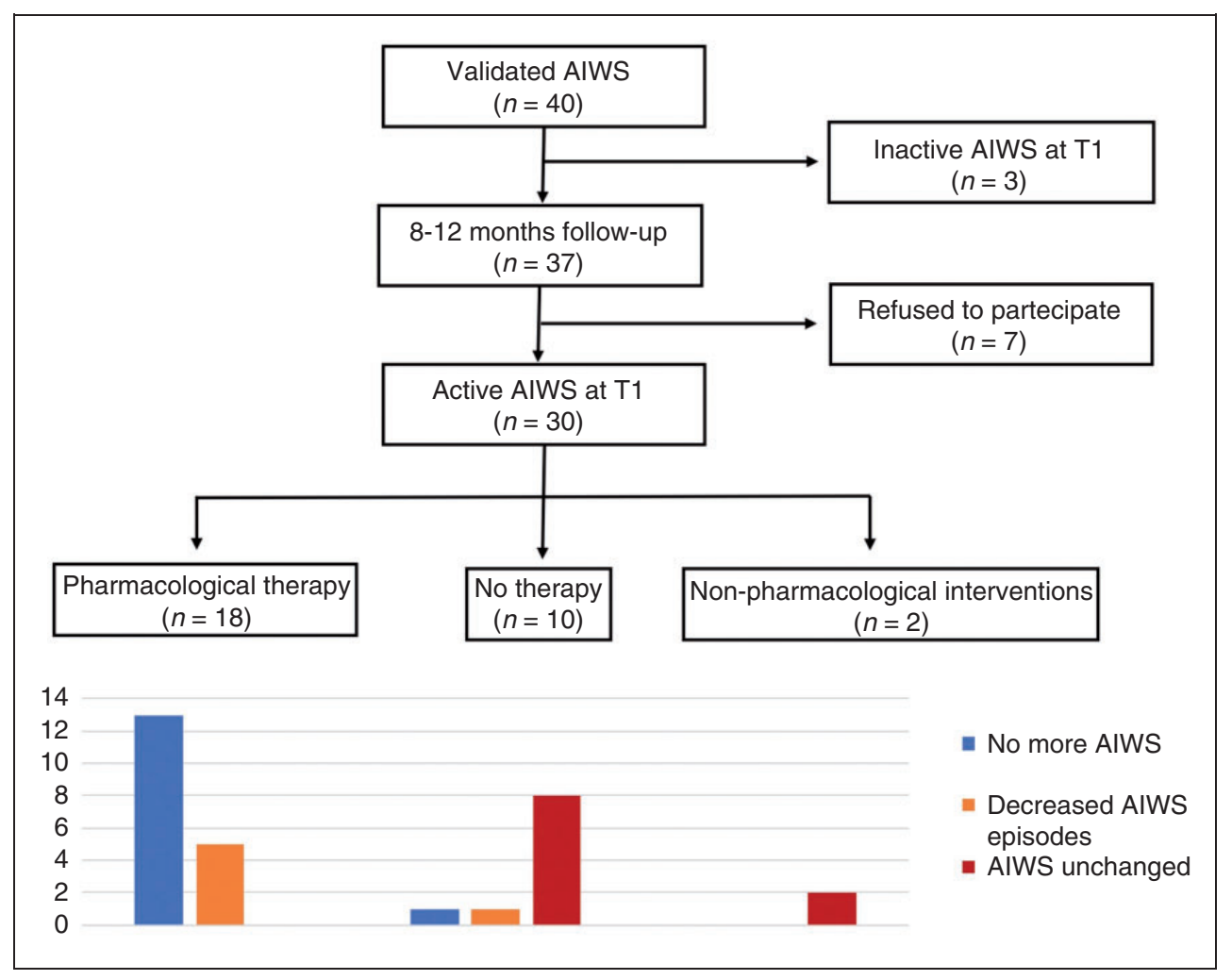

Figure 4. The figure displays a comprehensive flow-chart of the follow-up, comprising three graphs at the bottom showing the change in the frequency of AIWS episodes at the time of the follow-up for each subgroup according to the treatment.

In non-hemiplegic auras, CSD originates in the occipital lobe and can propagate to parietal cortices, causing, in a typical temporal order, scotomas, phosphenes and paresthesia. Similarly to migraine with aura, which rarely does not include visual symptoms $(34,35)$, in our cohort we did not find any patient with AIWS reporting pure somatosensory disturbances.

AIWS has been previously associated with derealisation and depersonalisation (5). Although we found a higher prevalence of both these disturbances among patients with AIWS, they were also present to a lesser extent in patients with a diagnosis of MA or MoA. In particular, there seems to be a continuum from migraineurs with AIWS to patients with MoA, with patients with MA having an intermediate clinical phenotype.

Likewise, depressive and anxiety disorders do not seem to be necessarily related to AIWS $(26,36,37)$, since we found no significantly different rate of depressive disorders and even lower prevalence of anxiety disorders in AIWS patients. This finding does not entirely exclude that depressive and anxiety disorders may play a role in the pathogenesis of AIWS, as suggested by previous reports $(21,26)$, but rather implies that these conditions are not sufficient per se to cause the symptoms in migraineurs.
Consistently with previous studies (20), our followup shows that pharmacological preventive therapy for migraine might be effective in reducing or completely resolving AIWS symptoms. On the other hand, differently from children, in which AIWS seems to be a selfterminating condition (38), we found that AIWS remained mostly unchanged in adult migraineurs who did not use treatment because of their relatively low migraine burden. Interestingly, most of the classes of medications generally used in migraine prevention such as antiepileptics, beta-blockers and antidepressants had a positive impact on AIWS. Most of these drugs have been proven to be effective on CSD $(39,40)$, further supporting the idea that CSD may underlie these symptoms in migraine.

Some limitations of the present study need to be taken into account. First, unfortunately, it was not possible to obtain follow-up information for all the patients. Yet, this is nonetheless the largest epidemiological study conducted on patients with migraine and AIWS so far. Secondly, a potential bias is that our data were collected in a dedicated headache unit; therefore, the prevalence of AIWS among non-help-seeking migraineurs in the general population might be different. However, as the characteristics of our sample (such 
as the prevalence of MA and MoA and chronic and episodic migraine) match those of larger population studies (1), even the prevalence of AIWS is likely to be representative of non-help-seeking migraineurs.

Finally, due to the relatively small sample of patients prescribed with preventive therapy, we could not test the efficacy of a specific class of medication. However, about half of the patients were treated with antiepileptic drugs, which are the class of medication that could most probably act on brain excitability. We hope that the high prevalence of AIWS demonstrated here may encourage future studies addressing this question.

In conclusion, we found that the prevalence of AIWS is higher than expected in adult migraineurs but in line with previous research in children and in particular subtypes of migraine. The relatively limited knowledge about this syndrome, which is still considered as a "rarity", could be related to a lack of communication between patients and the treating physicians. AIWS is predominantly associated to MA, and AIWS episodes invariantly produce visual disturbances, suggesting that in migraineurs AIWS may be the consequence of CSD. Lastly, since conventional migraine preventive therapy showed a positive effect on AIWS episodes, we strongly encourage clinicians to investigate the presence of AIWS episodes and to suggest the use of these drugs in those patients with recurrent and distressing episodes of AIWS, regardless of the burden of migraine itself.

\section{Key findings}

- The prevalence of AIWS in migraineurs is around $20 \%$ and doubles when considering only migraine with aura (MA), therefore AIWS should not be considered as a rare disorder.

- The underestimation of AIWS prevalence might be related to the unwillingness of patients to refer their symptoms to clinicians.

- Migraine preventive therapy might be effective in reducing the number of, and even resolving, AIWS episodes.

- The strong association between AIWS and MA and the responsiveness to migraine preventive therapy suggests a causal role of cortical spreading depression (CSD).

\section{Declaration of conflicting interests}

The authors declared no potential conflicts of interest with respect to the research, authorship, and/or publication of this article.

\section{Funding}

The authors received no financial support for the research, authorship, and/or publication of this article.

\section{ORCID iD}

Valentina Mancini (D) https://orcid.org/0000-0003-4411-896X

\section{References}

1. Goadsby PJ, Holland PR, Martins-Oliveira M, et al. Pathophysiology of migraine: A disorder of sensory processing. clinical manifestations neural basis of migraine triggers. Physiol Rev 2017; 97: 553-622.

2. Jürgens TP, Schulte LH and May A. Migraine trait symptoms in migraine with and without aura. Neurology 2014; 82: 1416-1424.

3. de Tommaso M, Ambrosini A, Brighina F, et al. Altered processing of sensory stimuli in patients with migraine. Nat Rev Neurol 2014; 10: 144-155.

4. Headache Classification Committee of the International Headache Society (IHS). The International Classification of Headache Disorders, 3rd edition (beta version). Cephalalgia 2013; 33: 629-808.

5. Todd J. The syndrome of Alice in Wonderland. Can Med J Assoc 1955; 73: 701-704.

6. Mastria G, Mancini V, Viganò A, et al. Alice in Wonderland Syndrome: A clinical and pathophysiological review. Biomed Res Int 2016; 2016: 8243145. DOI: $10.1155 / 2016 / 8243145$

7. Toole PO and Modestino EJ. Alice in Wonderland Syndrome: A real life version of Lewis Carroll's novel. Brain Dev 2017; 39: 470-474.

8. Entezami P, Paul A, Adamo MA, et al. Transient episode of Alice in Wonderland Syndrome after ventriculoatrial shunt revision. World Neurosurg 2018; 121: 149-151.

9. Mancini V, Mastria G, Frantellizzi V, et al. Aripiprazoletriggered Alice in Wonderland Syndrome episodes studied with 99mTc-HMPAO brain SPECT. Eur Neurol 2018; 79: 333-334.

10. Mastria G, Mancini V, Viganò A, et al. Temporal occipital glioblastoma presenting with Alice in Wonderland Syndrome in a patient with a long-time history of migraine without aura. Neurocase 2019; 24: 242-244.

11. Kadia BM, Ekabe CJ and Agborndip E. Primary care challenges of an obscure case of "Alice in Wonderland" syndrome in a patient with severe malaria in a resourceconstrained setting: A case report. BMC Infect Dis 2017; 17: $1-4$. 
12. Wang A. Alice In Wonderland Syndrome. In: Emergency Neuro-ophtalmology. Springer. 2018, pp. 297-299.

13. Palacios-Sánchez L, Botero-Meneses JS, GuerreroNaranjo A, et al. Alice in Wonderland Syndrome (AIWS). A reflection Síndrome de Alicia en el país de las maravillas. Revista Colombiana de Anestesiologia 2018; 46: 143-147.

14. Matsuura K, Ishikura R, Oguri M, et al. Atypical symptoms in migraine-related Alice in Wonderland Syndrome: Expansion of the phenotype and reflection on the pathomechanism. Yonago Acta Medica 2019; 62: 163-165.

15. Fine E, Farooq O, Finnegan $S$, et al. Alice in Wonderland Syndrome: Case series and analysis (P4.6030). Neurology 2019; 92: P4.6-030.

16. Lanska JR and Lanska DJ. Alice in Wonderland Syndrome: Somesthetic vs. visual perceptual disturbance. Neurology 2013; 80: 1262-1264.

17. Liu AM, Liu JG, Liu GW, et al. Alice in Wonderland Syndrome: Presenting and follow-up characteristics. Pediatr Neurol 2014; 51: 317-320.

18. Valenca MM, de Oliveira DA and de 1 Martins HA. Alice in Wonderland Syndrome, Burning Mouth Syndrome, cold stimulus headache, and HaNDL: Narrative review. Headache 2015; 55: 1233-1248.

19. Dooley JM, Bch MB, Hayley BAO, et al. Alice in Wonderland and other migraine associated phenomena - evolution over 30 years post headache diagnosis. Pediatr Neurol 2014; 51: 321-323.

20. Beh SC, Masrour S, Smith SV, et al. Clinical characteristics of Alice in Wonderland syndrome in a cohort with vestibular migraine. Neurol Clin Prac 2018; 8: 1-9.

21. Jan Dirk B. Alice in Wonderland Syndrome. Neurol Clin Pract 2016; 6:259-270.

22. Podoll K, Ebel H, Robinson D, et al. Obligatory and facultative symptoms of the Alice in Wonderland Syndrome. Minerva Med 2002; 93: 287-293.

23. Lo Buono V, Bonanno L, Corallo F, et al. Cognitive functions and psychological symptoms in migraine: A study on patients with and without aura. Int $J$ Neurosci 2018; 129: 588-592.

24. Vuralli D, Ayata C and Bolay H. Cognitive dysfunction and migraine. J Headache Pain 2018; 4: 109.

25. Minen MT, De Dhaem OB, Van Diest AK, et al. Migraine and its psychiatric comorbidities. J Neurol Neurosurg Psychiatry 2016; 87: 741-749.

26. Eric B, Aurelien C and Laurent S. Alice in Wonderland Syndrome in major depressive disorder. $J$ Neuropsychiatry Clin Neurosci 2010; 22: 352j.e16-352. e16.
27. Yokoyama T, Okamura T, Takahashi M, et al. A case of recurrent depressive disorder presenting with Alice in Wonderland Syndrome: Psychopathology and pre- and post-treatment FDG-PET findings. BMC Psychiatry 2017; 17: 4-9.

28. May A and Schulte LH. Chronic migraine: Risk factors, mechanisms and treatment. Nat Rev Neurol 2016; 12: 455-464.

29. Lampl C, Thomas H, Tassorelli C, et al. Headache, depression and anxiety: Associations in the Eurolight project. J Headache Pain 2016; 17: 59.

30. Buse DC, Reed ML, Fanning KM, et al. Comorbid and co-occurring conditions in migraine and associated risk of increasing headache pain intensity and headache frequency: Results of the Migraine in America Symptoms and Treatment (MAST) study. J Headache Pain 2020; 21 : 23.

31. Petolicchio B, Squitieri M, Viganò A, et al. Psychodynamic functioning in chronic headache patients: A short term psychodynamic psychotherapy (STPP) study. J Headache Pain 2015;16: 1-2.

32. Altieri M, Di Giambattista R, Di Clemente L, et al. Combined pharmacological and short-term psychodynamic psychotherapy for probable medication overuse headache: A pilot study. Cephalalgia 2009; 29: 293-299.

33. Charles AC and Baca SM. Cortical spreading depression and migraine. Nat Rev Neurol 2013; 9: 637-644.

34. Viana M, Linde M, Sances G, et al. Migraine aura symptoms: Duration, succession and temporal relationship to headache. Cephalalgia 2015; 36: 413-421.

35. Russell MB and Olesen J. A nosographic analysis of the migraine aura in a general population. Brain 1996; 119: 355-361.

36. Mizuno $M$, Kashima $H$, Chiba $H$, et al. 'Alice in Wonderland' Syndrome as a precursor of depressive disorder. Psychopathology 1998; 160: 85-89.

37. Rasmussen PD, Vilmar JW and Nielsen JEK. Alice in Wonderland-syndrome may be confused with anxiety. Ugeskr Laeger 2019; 181: 31280762.

38. Weidenfeld A and Borusiak P. Alice-in-Wonderland syndrome - a case-based update and long-term outcome in nine children. Childs Nerv Syst 2011; 27: 893-896.

39. Ayata C, Jin H, Kudo C, et al. Suppression of cortical spreading depression in migraine prophylaxis. Ann Neurol 2006, 59: 652-661.

40. Bogdanov V, Multon S, Chauvel V, et al. Migraine preventive drugs differentially affect cortical spreading depression in rat. Neurobiol Dis 2011; 41: 430-435. 